\title{
A REPRESENTATION CHARACTERIZATION THEOREM
}

\author{
WILLIAM D. L. APPLING
}

ABSTRACT. Given a field $\mathbf{F}$ of subsets of a set $U$ and a real. valued function $T$ defined on a set $S$ of functions from $F$ into $\exp (R)$ with bounded range unions, a necessary and sufficient condition is given in order that there be a bounded finitely additive function $\theta$ from $\mathbf{F}$ into $\mathbf{R}$ such that if $\alpha$ is in $S$, then the integral $\int_{U} \alpha(I) \theta(I)$, as a variational integral, i.e., a refinement-wise limit of appropriate sums over (finite) subdivisions, exists and is $T(\alpha)$.

1. Introduction. Suppose $U$ is a set, $\mathbf{F}$ is a field of subsets of $U, p$ is the set of all functions from $F$ into $\exp (\mathbf{R}), p_{B}$ is the set of all elements of $\mathfrak{p}$ with bounded range union, $\mathfrak{p}_{A B}$ is the set of all bounded finitely additive functions from $F$ into $R$, and $\mathfrak{p}_{A}^{+}$is the set of all nonnegative-valued elements of $p_{A B^{\circ}}$

Suppose $S \subseteq p_{B}$ and $T$ is a function from $S$ into $R$. In this paper we establish a necessary and sufficient condition that $T$ have a certain kind of "integral representation" (see below) with respect to some element of $p_{A B}$; specifically, we demonstrate the following characterization theorem $(\S 3)$ :

Theorem 3.1. If $S \subseteq p_{B}$ and $T$ is a function from $S$ into $\mathbf{R}$, then the following two statements are equivalent:

(1) there is $\theta$ in $P_{A B}$ such that if $\alpha$ is in $S$, then the integral (see $\$ 2$ ) $\int_{U} \alpha(I) \theta(I)$ exists and is $T(\alpha)$; and

(2) there is a linear subspace $S^{\prime}$ of $p_{B}$, a function $P$ from $S^{\prime}$ into the nonnegative numbers, and a real linear functional $T^{\prime}$ on $S^{\prime}$ such that:

(i) $T \subseteq T^{\prime}$,

(ii) $\left\{T^{\prime \prime}\left(\chi_{V}\right): V\right.$ in $\left.\mathbf{F}\right\}$ is bounded, where for each $V$ in $\mathbf{F}, \chi_{V}$ is in $S^{\prime}$ and is defined by:

$$
\chi_{V}(I)= \begin{cases}\{1\} & \text { if } I \subseteq V \\ \{0\} & \text { otherwise, }\end{cases}
$$

Presented to the Society, February 6, 1974; received by the editors May 15,1974. AMS (MOS) subject classifications (1970). Primary 28A45; Secondary 46E99. tation.

Key words and phrases. Set function, variational integral, functional represen- 
(iii) $T^{\prime}(\alpha) \rightarrow 0$ as $P(\alpha) \rightarrow 0$, $\alpha$ in $S^{\prime}$, and

(iv) if $a$ is in $S^{\prime}$ and $0<c$, then there is a subdivision (see $\$ 2$ ) ? of $U$ such that if $(\xi$ is a refinement (see $\$ 2)$ of $\supseteqq$ and for each $V$ in $($, $a(V)$ is in $\alpha(V)$, then $P\left(\alpha-\Sigma_{\Subset} a(V) \chi_{V}\right)<c$.

2. Preliminary theorems and definitions. We refer the reader to [3] for the notions of subdivision, refinement and integral used in this paper. We shall let "( $<<$ )" mean "F is a refinement of $\mathscr{D}$ ". We also refer the reader to [3] for a statement of Kolmogoroff's [5] notion of differential equivalence and its implications about the existence and equivalence of the integrals that we shall use. In this paper, when the existence of an integral or the equivalence of an integral to an integral is an easy consequence of the above mentioned material or the material of this section, the integral need simply be written or the equivalence assertion made, and the proof of existence or equivalence left to the reader.

We state two extensions of previous interval function theorems of the author. The proofs carry over with only minor modifications.

Theorem 2.A.1 (see [1] for the interval function version). If $\alpha$ is in $\mathfrak{p}_{B}$ and $\theta$ is in $\mathfrak{p}_{A B^{\prime}}$, then $\int_{U} \alpha(I) \theta(I)$ exists iff $\int_{U} \alpha(I) \int_{I}|\theta(J)|$ exists.

Theorem 2.A.2 (see [2] for the interval function version). If $\alpha$ is in $\mathfrak{p}_{B}, \theta$ is in $\mathfrak{p}_{A B}$, and $\int_{U} \alpha(I) \theta(I)$ exists, then $\int_{U}|\alpha(I)| \theta(I)$ exists.

We end this section by stating a special form of the "Lipschitz portion" of the Bochner-Radon-Nikodým theorem that we shall use in the proof of Theorem 3.1.

Theorem 2.A.3 [4]. If $\alpha$ is in $\mathfrak{p}_{B}, \mu$ is in $p_{A}^{+}$and $\int_{U} \alpha(I) \mu(I)$ exists, then

$$
\int_{U}\left[\int_{V}|\alpha(V)-\alpha(I)| \mu(I)\right]=0
$$

i.e., if $0<c$, then there is $\mathscr{S}<<\{U\}$ such that if $\mathbb{E}<<\mathscr{S}$ and for each $V$ in $\mathbf{F}, a(V)$ is in $\alpha(V)$, then

$$
\sum_{\varepsilon} \int_{V}|a(V)-\alpha(I)| \mu(I)<c .
$$

3. The representation characterization theorem. In this section we prove Theorem 3.1, as stated in the Introduction. 
Proof. Suppose (2) is true. Let $\theta$ be the function from $\mathbf{F}$ into $\mathbf{R}$ defined by:

$$
\theta(V)=T^{\prime}\left(\chi_{V}\right)
$$

Clearly $\theta$ is bounded.

We now show that $\theta$ is finitely additive. Suppose $V$ and $W$ are two mutually exclusive sets of $\mathbf{F}$ and $0<c$.

There is $d>0$ such that if $\alpha$ is in $S^{\prime}$ and $P(\alpha)<d$, then $\left|T^{\prime}(\alpha)\right|<$ $c / 2$. There is $\mathscr{S}<<\{V, W, U-[V \cup W]\}$ such that if $\mathbb{E} \ll \mathscr{S}$, and for each $I$ in $\mathbb{E}, a(I)$ is in $\chi_{V \cup W}$ and $b(I)$ is in $\left[\chi_{V}+\chi_{W}\right](I)$, then

$$
\max \left\{P\left(\chi_{V \cup W}-\sum_{\mathfrak{E}} a(I) \chi_{I}\right), P\left(\chi_{V}+\chi_{W}-\sum_{\mathfrak{G}} b(I) \chi_{I}\right)\right\}<d .
$$

Suppose $I$ is in $\mathscr{I}$. Either $I \subseteq V, I \subseteq W$, or $I \subseteq U-[V \cup W]$. Therefore

$$
\left[\chi_{V}+\chi_{W}\right](I)= \begin{cases}\{1\} & \text { if } I \subseteq V \cup W, \\ \{0\} & \text { otherwise, }\end{cases}
$$

and since

$$
\chi_{V \cup W}(I)= \begin{cases}\{1\} & \text { if } I \subseteq V \cup W \\ \{0\} & \text { otherwise }\end{cases}
$$

it follows that

$$
\chi_{V \cup W}(I)=\left[\chi_{V}+\chi_{W}\right](I)
$$

Therefore for each $I$ in $\mathscr{S}$ there is $c(I)$ in both $\chi_{V \cup W}(I)$ and $\left[\chi_{V}+\chi_{W}\right](I)$, so that

$$
\max \left\{P\left(\chi_{V \cup W}-\sum_{\mathcal{D}} c(I) \chi_{I}\right), F\left(\chi_{V}+\chi_{W}-\sum_{\mathcal{D}} c(I) \chi_{I}\right)\right\}<d,
$$

which implies that

$$
\max \left\{\left|T^{\prime}\left(\chi_{V U W}-\sum_{\mathcal{D}} c(I) x_{I}\right)\right|,\left|T^{\prime}\left(\chi_{V}+\chi_{W}-\sum_{\mathcal{D}} c(I)_{X_{I}}\right)\right|\right\}<\frac{c}{2},
$$

which, together with the linearity of $T^{\prime}$, implies that $\mid T^{\prime}\left(\chi_{V \cup W}\right)-$ $T^{\prime}\left(\chi_{V}+\chi_{W}\right) \mid<c$.

Therefore

$$
\theta(V \cup W)=T^{\prime}\left(\chi_{V \cup W}\right)=T^{\prime}\left(\chi_{V}+\chi_{W}\right)=T^{\prime}\left(\chi_{V}\right)+T^{\prime}\left(\chi_{W}\right)=\theta(V)+\theta(W) .
$$

Therefore $\theta$ is finitely additive. 
Now suppose $\alpha$ is in $S^{\prime}$. Suppose $0<c$. There is $d>0$ such that if $\beta$ is in $S^{\prime}$ and $P(\beta)<d$, then $\left|T^{\prime}(\beta)\right|<c$. There is $\mathscr{S} \ll\{U\}$ such that if $\mathbb{E}<\mathcal{I}$ and for each $I$ in $\mathfrak{F}, a(I)$ is in $\alpha(I)$, then $P\left(\alpha-\Sigma_{\mathbb{E}} a(I) \chi_{I}\right)<d$, so that

$c>\left|T^{\prime}\left(\alpha-\sum_{\mathfrak{G}} a(I) \chi_{I}\right)\right|=\left|T^{\prime}(\alpha)-\sum_{\mathfrak{C}} a(I) T^{\prime}\left(\chi_{I}\right)\right|=\left|T^{\prime}(\alpha)-\sum_{\mathfrak{G}} a(I) \theta(I)\right|$.

Therefore $\int_{U} \alpha(I) \theta(I)$ exists and is $T^{\prime}(\alpha)$.

Since $T \subseteq T^{\prime}$, we see that (2) implies (1).

Now suppose (1) is true. Let $S^{\prime}$ denote the set to which $\beta$ belongs iff $\beta$ is in $p_{B}$ and $\int_{U} \beta(I) \theta(I)$ exists. Clearly $S^{\prime}$ is a linear space. Let $P$ and $T^{\prime}$ be functions defined on $S^{\prime}$ respectively by:

$$
P(\beta)=\int_{U}|\beta(I)| \int_{I}|\theta(J)| \text { and } T^{\prime}(\beta)=\int_{U} \beta(I) \theta(I) .
$$

Clearly condition (i) of (2) holds, and from the inequality, for each $\beta$ in $S^{\prime}$,

$$
\left|T^{\prime}(\beta)\right| \leq P(\beta) \leq \sup \{x \mid x \text { in range union of } \beta\} \int_{U}|\theta(I)|,
$$

we easily see that (ii) and (iii) of (2) are satisfied. Now, suppose $\alpha$ is in $S^{\prime}$ and $0<c$. By Theorem $2 . A .3$ there is $I<<\{U\}$ such that if $\mathscr{S}<<$ and for each $V$ in $\mathbb{F}, a(V)$ is in $\alpha(V)$, then

$$
\sum_{\mathbb{G}} \int_{V}|a(V)-a(I)| \int_{I}|\theta(J)|<c,
$$

so that letting $W$ and $V$ both denote elements of $(\xi$, we have

$$
\begin{aligned}
P\left(\alpha-\sum_{\mathfrak{E}} a(V) \chi_{V}\right) & =\int_{U}\left|\alpha(I)-\sum_{\mathfrak{E}} a(V) \chi_{V}(I)\right| \int_{I}|\theta(J)| \\
& =\sum_{\mathfrak{G}} \int_{W}\left|\alpha(I)-\sum_{\mathfrak{E}} a(V) \chi_{V}(I)\right| \int_{I}|\theta(J)| \\
& =\sum_{\mathfrak{G}} \int_{W}\left|\alpha(I)-a(W) \chi_{W}(I)\right| \int_{I}|\theta(J)| \\
& =\sum_{\mathfrak{E}} \int_{W}|\alpha(I)-a(W)| \int_{I}|\theta(J)|<c .
\end{aligned}
$$

Therefore (iv) of (2) is satisfied.

Therefore (1) and (2) are equivalent.

\section{REFERENCES}

1. W. D. L. Appling, Interval functions and the Hellinger integral, Duke Math. J. 29 (1962), 515-520. MR $25 \# 4075$. 
2. W. D. L. Appling, Interval functions and continuity, Rend. Circ. Mat. Palermo (2) 11 (1962), 285-290. MR 29 \#3600.

3. - Summability of real-valued set functions, Riv. Mat. Univ. Parma (2) 8 (1967), 77-100. MR 40 \#4418.

4. - Set functions, finite additivity and distribution functions, Ann. Mat. Pura Appl. 96 (1973), 265-287.

5. A. Kolmogoroff, Untersuchungen über den Integralbegriff, Math. Ann. 103 (1930), 654-696.

DEP ARTMENT OF MATHEMATICS, NORTH TEXAS STATE UNIVERSITY, DENTON, TEXAS 76203 